\title{
LA REVISIÓN DE UN CUENTO MEDIEVAL EN UN SITIO DE INTERNET
}

\author{
Monique Noël-Gaudreault \\ Flore Gervais
}

"Veinte veces sobre el telar ponga su obra... » Nicolas Boileau

El presente artículo tiene por tema la revisión de texto con la ayuda de un sitio Internet. Nuestrapreguntade investigación es la siguiente: “¿Cuáles serian los componentes de la fase de revisión de una secuencia didáctica cuyo tema es la producción escrita, con soporte informático, de un cuento medieval?».

Además de las ventajas de un sitio Internet presentamos los conceptos necesarios para la relectura y reescritura de un texto: la integralidad del texto, la coherencialcohesión, el vocabulario, la sintaxis y la ortografía.
This article has as a topic the revision of a text with the help of a Web page. Our question was, "What are the elements of the rewriting phase for a didactical sequence on fiction writing, when using computer tools, in this case to create a tale within a medieval setting?"

We summarize the advantages of using a Web site for this, and we go on by tracing out the concepts that underlie rereading and rewriting the tale, and by listing the main objects to check during the revision process, completeness, coherence-cohesion, vocabulary; syntax and spelling.

* Ph.D. en literatura de la Universidad Laval, Québec, Canada (1988). Professora asociada de la Facultad de Ciencias de la Educación de la misma universidad. Ha participado en diversas actividades internacionales: conferencias en Nijni Novgorod: (conferenciante invitada, 1995, 1996), Estados Unidos: CIEF (Conseil international d'études francophones, 1998, 1999, 2001, 2003), Paris: FIPF (Federation internacionale des professeurs de français, 2000, 2001, 2002, 2003), Tokyo FIPF. 1996). ( México: AFIRSE (Association francophone internationale de recherche en sciences de l'éducation, 1999).

** Ph.D. en linguística de la Universidad de Montréal, Québec, Canada (1974). Professora titular de la Facultad de Ciencias de la Educación de la misma universidad. Ha participado en diversas actividades internacionales: conferencias en los Estados Unidos: CIEF (Conseil international d'études francophones, 1998, 1999, 2001, 2003). Paris: FIPF (Federation internacionale des professeurs de français, 2000), Toulouse: Symposium international du REF (Réseau de recherche en éducation et formation, 1998), México: AFIRSE (Association francophone internationale de recherche en sciences de l'éducation . 1999), Cuba (Pedagogía 99). 
Los escritores novatos piensan que los textos que escriben dicen exactamente aquello que ellos querían decir. Por consecuencia, la revisión les parece inútil (Daiute y Kruidenier,1985).

En estas condiciones, es comprensible que tener que revisar el texto sea. para ciertos alumnos. sinónimo de fracaso y, para otros, una sanción.

En efecto, cuando se les solicita revisar, no saben qué hacer. Ignoran cómo descentrarse, es decir, cómo tomar el lugar de un lector potencial. Esta lectura crítica, necesaria, tiene un doble objetivo:

a. corregir las imperfecciones del texto inicial

b. mejorar las informaciones ya escritas en el texto. (Bourque, Nöel-Gaudreault y otros, 1987).

Es cierto que la revisión es una habilidad difícil de adquirir y que ella constituye uno de los obstáculos de la enseñanza de la escritura (Joram y otros,1992). Por esta razón, incumbe a los especialistas en didáctica encontrar medios para facilitar a los escritores novatos más control sobre el proceso de revisión de texto y, a la vez, sobre el proceso de producción.

Nuestro equipo de investigación, subvencionado en el marco del programa"Fondos para la Formación de Investigadores y de Ayuda a la investigación"(FCAR), está elaborando un sitio de Internet en didáctica de la producción escrita. Sólo nos ocuparemos en el presente artículo de la revisión de texto.

¿Cuáles serían entonces los componentes de la fase de revisión de una secuencia didáctica relativa a la producción escrita de un cuento medieval en soporte informático (sitio de Internet)? En este artículo presentaremos, en primer lugar, los conceptos subyacentes a la revisión, luego expondremos los componentes de la didáctica de la revisión y, por último, describiremos las características de un medio informático.

\section{La revisión: conceptos subyacentes}

Los conceptos de la revisión son los siguientes: proceso de escritura, recursividad, relectura, reescritura. Luego de presentar estos conceptos, intentaremos definir qué es un escritor experto, es decir, aquel que domina la competencia de la revisión.

Proceso de escritura. Saber redactar implica la capacidad de tomar en cuenta simultáneamente la búsqueda de ideas, la planificación, la redacción y la revisión, tanto en el aspecto discursivo como lingüístico. Para los escritores 
novatos, esto implica una sobrecarga cognitiva (Graves, 1983), la que sólo se aligerará con estrategias eficaces y un entrenamiento de tipo deportivo. ¡Al menos eso esperamos!

Recursividad. Los procesos de escritura sobre los que Hayes y Flower (1980) trabajaron se caracterizan por el hecho de poder repetirse de forma indefinida y porque cada una de las etapas ("processes"), si las hay, puede ser gestionada junto con otras: por ejemplo, la búsqueda de ideas puede intervenir nuevamente una vez realizada la redacción de la primera versión del texto. Para los expertos, si cada etapa -cronológicamente- constituye un momento importante en un instante determinado, ella no está jamás exenta de ser retomada o de anticipaciones.

Relectura. La relectura del texto producido, ya sea al final de la producción o a lo largo del proceso de escritura, exige un distanciamiento del escritor respecto a los trazos que ya ha dejado (Fayol y Schneuwly,1987). Para el escritor, esta relectura (constituida, de hecho, de múltiples relecturas) consiste en retomar el hilo de sus ideas, en detenerse y en anticipar la continuación del trabajo. Ella apunta a mejorar el producto comparando dos representaciones: la del texto, tal como él debería ser en función del referente (temática por tratar), del objetivo buscado y del destinatario al cual se dirige, y la del texto que se tiene a la vista. Tal comparación puede efectuarse muchas veces a lo largo del proceso de escritura.

Reescritura. Reescribir el texto producido consiste en anotar observaciones sobre el mismo en los márgenes de la página y en hacer correcciones. La reescritura involucra las palabras, las proposiciones, las frases, el texto completo. Hay modificaciones que no cambian el sentido global del texto y otras que sí lo hacen. En CDO (Compare, Diagnosis, Operate) de Scardamalia y Bereiter (1986), la reescritura corresponde a"Operate", es decir, operar las modificaciones que se juzgan necesarias. Estas pueden ser clasificadas esquemáticamente en cuatro categorías: incorporaciones o agregados, eliminaciones o exclusiones, sustituciones o reemplazos, reordenamientos o reubicaciones (Fabre, 1989).

Competencia de la revisión. El escritor experto puede representarse el texto por producir, centrarse sobre lo que ha producido y ver las mejoras que podría aportar a la organización global de dicho texto (Roussey, Farioli y Piolat, 1994). Lejos de detenerse en retoques"locales", el escritor experto consagra lo esencial de su tiempo a elaborar ideas nuevas o a mejorar aquellas que ya figuran en el papel. En breve, el experto realiza una revisión más profunda, hace más retoques sobre la frase y el texto. (Scardamalia y Bereiter, 1986). 


\section{El entorno informático: cualidades buscadas.}

A nuestro entender, un sitio de Internet para la producción de un cuento medieval debería tener las mismas cualidades que un material didáctico informático: de fácil utilización, rendimiento, control, polivalencia, fiabilidad y cualidades estéticas (Gingras y Loiselle, 1990).

Un material didáctico informático es"de fácil utilización"cuando es fácilmente utilizable por personas no especializadas.

El rendimiento se mide en función de la aptitud de ejecutar una tarea de manera relativamente rápida y eficaz..

En cuanto al control, podemos definirlo como la capacidad del sitio para verificar la ejecución de las tareas, o al menos para guardar en la memoria las operaciones efectuadas por el usuario para permitir un retorno y un diálogo profesor-alumno sobre dichas operaciones.

La polivalencia es sinónimo de flexibilidad, apertura, adaptabilidad...

Idealmente, la fiabilidad representa, para todo propósito, la capacidad de funcionar sin fallas durante un determinado período y bajo las condiciones previstas.

Por último, las cualidades estéticas están destinadas a alumnos de primaria y de secundaria y no representan un lujo sino una necesidad: el atractivo visual puede sostener la motivación que, como se sabe, es una condición sine qua non de éxito del aprendizaje.

\section{Didáctica de la revisión con las TIC}

Las TIC (Tecnologías de Información y Comunicación) permiten también más interacciones entre el escritor y el texto en elaboración. La distancia y la nitidez de la impresión del texto permiten que el ojo realice una exploración más sistemática sobre los objetos textuales por revisar ¿Cuáles?

\section{Los aspectos de la revisión}

Los aspectos de la revisión son, en orden, la integralidad, la coherencia, el vocabulario, la sintaxis, la ortografía y la gramática.

\section{La integralidad (del texto)}

En didáctica, el concepto de integralidad remite a una doble problemática:

a. la de la evaluación, ligada a la pregunta:"¿Qué es un buen texto de alumno?” 
b. la del mejoramiento, ligada a la pregunta:"Dado un texto x producido por un alumno, ¿cómo hacer para ayudarlo a detectar los problemas de carencias o faltantes y a remediarlos?"

Un relato completo es el que respeta los elementos invariables de la estructura narrativa. Para Aristóteles, una historia tiene una presentación, un nudo y un desenlace. Es un proceso cerrado. Desde la antigüedad, se han multiplicado las teorías y los teóricos; se llegó al consenso de que los eventos se organizan en esquemas. Cualquiera que sea el nombre dado a las etapas observadas, un evento es lo que nos lleva a contar algo (Vanderdope, 1995). Esto implica la modificación de un estado estable desde un cierto tiempo, seguida de la acción de un personaje que reacciona contra la nueva situación y decide resolver el problema. Un relato completo lleva generalmente al lector o al oyente hacia la resolución del problema.

Hay, ciertamente, informaciones necesarias para que la historia forme un todo. Sin embargo, ese todo puede variar en extensión: el relato puede ser más o menos detallado (Laib , 1990). Una vez que se definió lo esencial, ¿cuántos detalles se deben incorporar?

¿Cuántos elementos adicionales se deben incorporar para sostener el interés del lector y, a la vez, considerar su capacidad de comprensión (inteligencia y memoria)?

En síntesis, la integralidad del texto no tiene nada que ver con la cantidad de información sino con el hambre de conocimiento o ansia de saber del lector. En consecuencia, es relativa a un lector o grupo determinado.

\section{La coherencia/cohesión}

Esta noción, útil aunque difícil de definir, es sinónimo de orden lógico, de homogeneidad, de compatibilidad y de incorporación de información. En didáctica, el concepto de coherencia/cohesión (Charolles,1978) está caracterizado por cuatro reglas, las cuales se resumen globalmente en cuatro cuestiones:

- ¿Hay elementos recurrentes en el desarrollo lineal del texto (reglas de repetición)?

- ¿Se desarrolla el tex to beneficiándolo de un aporte semántico constantemente renovado? (regla de progresión)

- ¿Se desarrolla el texto sin introducir ningún elemento semántico que contradiga un contenido planteado, admitido anteriormente o deducible de éste por inferencia? 
- ¿Denota el texto hechos relacionados entre ellos en el mundo representado? o ¿es posible vincular dos frases consecutivas por medio de un conector? (regla de relación)

Las siguientes reglas nos ayudarán a esclarecer lo antedicho:

Un texto es coherente si:

- contiene informaciones que presentan cierta unidad temática.

- se enriquece con un aporte renovado de informaciones

- no contiene elementos contradictorios o inverosímiles

- presenta informaciones ligadas entre sí.

En síntesis, la coherencia/cohesión se puede evaluar con cierta objetividad aunque, en algunos casos, se superponga a la integralidad.

\section{El vocabulario}

¿Cómo se puede ayudar a los alumnos a detectar y resolver los problemas de vocabulario en sus textos?

Se considera que un vocabulario es adecuado cuando los términos utilizados expresan, con la mayor exactitud posible, lo que el escritor quiere decir. (Tompkins, 1992)

Nosotros consideramos que un vocabulario es rico cuando le da al lector la posibilidad de ver, de sentir, de degustar grupos de palabras. En pocas palabras, cuando escapa del lenguaje"de base cotidiana".

El vocabulario es no repetitivo, cuando existe diversidad o variedad de palabras, las que hacen que un texto sea un buen texto ( salvo en la poesía, por supuesto, en la que la repetición es considerada una estrategia de escritura).

\section{La sintaxis}

Diremos que la sintaxis de un texto es correcta si respeta las reglas de construcción de una frase, sea ésta simple o compleja.

Consideraremos que la sintaxis de un texto es variada cuando las frases que lo componen no obedecen todas a la misma estructura de tipo sujeto-verbocomplemento (Samway, 1987). 


\section{La ortografía}

Sometidas a reglas estrictas, ni la ortografía ni la gramática presentan verdaderamente problemas de evaluación. Sin embargo, la revisión le ofrece al escritor, ahora en el rol de lector crítico, diversas posibilidades: de cuestionarse, de discutir con sus pares, de activar la metacognición.

\section{El soporte informático: ¿Cómo?}

\section{La utilización de las TIC para el proceso de revisión}

Para los estudiantes, las TIC estimulan su curiosidad, desarrollan su capacidad de reflexión y de resolución de problemas así como su capacidad para localizar, reagrupar y seleccionar información. Las TIC favorecen la creatividad, apelan a la atención, al rigor intelectual, a la precisión de gestos; en fin, posibilitan la autocorrección. (Dwyer y otros 1997; Versini, 1996; Joram y otros, 1992).

Un medio informático puede simplificar la tarea de revisión. Placer y facilidad se dan cita: $¡$ No es necesario volver a copiar el texto una vez decididos los cambios que se realizarán!

Para llevar a cabo la fase de revisión de un cuento medieval, las tecnologías permiten tornar más disponibles y accesibles, a la vez, los herramientas o instrumentos y las consignas de la revisión.

\section{Las herramientas de la revisión}

Existen dos tipos de herramientas de revisión que permiten escribir más y revisar más: las fichas y las rejillas (llamadas también "grillas"). Las fichas proporcionan principalmente listas de las cuales los escritores podrán sacar provecho: por ejemplo, listas de palabras relacionadas, listas de palabras para sustituir palabras vulgares, sin precisión, listas de palabras o expresiones para matizar las emociones y los sentimientos, listas de información sobre la edad media (campesinos, nobles, clérigos,...)

El lector encontrará en el anexo dos ejemplos de fichas de revisión: "Cómo designar al personaje principal" y "Cómo eliminar las palabras vulgares". Estas ofrecen soluciones a los dos problemas más frecuentes que se presentan al revisar el contenido de los textos de los escritores aprendices.

Nos falta elaborar rejillas para facilitar el auto-cuestionamiento sobre los diversos aspectos a examinar, es decir, la integralidad (o cantidad suficiente de informaciones relativas al esquema del texto, a los personajes, a los lugares, a 
los objetos): la coherencia (según las cuatro reglas de Charolles (1978)): la sintaxis (correcta y variada): el vocabulario (adecuado, rico y no repetitivo): la ortografía y la gramática (correcta).

\section{Las consignas de revisión}

Sin embargo, estas herramientas serían de poca utilidad sin las consignas de revisión que apuntan a indicar cómo proceder y en qué orden temporal. Es aconsejable que un colega realice una relectura a fin de formular observaciones críticas y convertir luego estas observaciones en consignas (García-Debanc:1996). Según esta autora, las lecturas críticas solicitan muchas informaciones complementarias, principalmente del orden de la descripción. Cada una de estas consignas constituye un criterio que podrá ser tomado en cuenta durante la evaluación del texto final.

\section{Conclusión}

Así como es difícil ayudar a los alumnos a escribir un texto, también la revisión de este mismo texto parece problemática, a falta de motivación y de estrategias.

Nosotros estamos elaborando un sitio de Internet para la producción de un cuento medieval. Esto supone la elaboración y prueba de herramientas didácticas de revisión relativas, especialmente, a la integralidad del texto, a la coherencia/ cohesión, al vocabulario, a la sintaxis así como a la ortografía y gramática. Pensamos que las TIC nos ayudarán a desarrollar la capacidad de reflexión de los alumnos con el objetivo de resolver los numerosos problemas que posee la escritura y, sobre todo, la revisión.

Nos falta espacio y tiempo para ir más lejos. Lo que es seguro, es que construir un sitio de Internet es más difícil cuando las nociones en cuestión son complejas y numerosas.

“El genio es una larga paciencia”... ¡Es el don que es necesario desear a nuestros alumnos!

\section{Referencias}

Bourque, G., Noel-Gaudreault, M. y otros, (1987). L'École à fictions. 3 vol. Québec (Canada), Presses de l'Université du Québec, 528 p.

Charolles, Michel (1978).'Introduction aux problèmes de la cohérence des textes". Langue française, no 38, Larousse, Paris. 
Daiute, Colette A. (1985). Writing and Computer. Reading MASS: Addisson Westey.

Daiute, Colette et Kruidenier. John (1985)"A self questioning strategy to increase young writers' revising processes."Applied psycholinguistique no 6. pp. 307-318.

Dwyer y otros (1997). La classe branchée: enseigner à l'ère des technologies. La Chenelière - McGraw Hill.

Fabre, C. (1989). L'usage didactique des brouillons: une pratique de diversification à construire. Dans J.-L. Chiss, J.-P. Laurent, J.-C. Meyer, H. Romian, B. Schneuwly (dir.) (1987). Apprendre/Enseigner à produire des textes écrits. Actes du 3 e colloque international de didactique du français Namur 09. 1986, Bruxelles, De Boeck Université, coll. Prisme, série Didactique, no. 3.

Fayol, M. y Schneuwly, B. (1987). La mise en texte et ses problèmes. Dans J.-L. Chiss, J.-P. Laurent, J.-C. Meyer, H. Romian, H.B. Schneuwly (dir.) (1987) Apprendre/Enseigner à produire des textes écrits. Actes du $3^{e}$ colloque international de didactique du français Namur 09-1986, Bruxelles, De Boeck Université, coll. Prisme, série Didactique, no 3.

Garcia-Debanc, Claudine (1996)."Consignes d'écriture et création", Pratiques no 89, pp. 69-88.

Gingras, Jacques R. y LOISELLE, Jean (1990) Guide d'évaluation des didacticiels, Québec, Ministère de l'Éducation du Québec.

Graves, D. (1983). Writing: Teachers and Children at Work. New Hampshire, Heineman Educational Books, Portsmouth, NH.

Hayes, John R. et Flower, Linda S. (1980). Identifying the organisation of writing process. In: W. Gregg et R. Steinberg, (eds), Cognitive processes in writing. Hillsdale-New Jersey: Lawrence Erlbaum Associates publishers, 33-50.

Joram, Elana; Woodruff, Earl; Bryson, Mary et Lindsay, Peter (1992)"The effects of revising with a word processor on written composition", Research in the teaching of English, vol 26, no 2, pp. 167-193. 
Laib. Nevin (1990)"Conciseness and Amplification". College Composition and Communication", vol 41 no 4, pp. 443-459.

Roussey, Jean-Yves; Farioli, F. y Piolat, Annie (1994)."Effects of social regulation and computer assistance on the monitoring of writing", European Journal of Psychology of Education, vol 7, no 4, pp. 235-309.

Scardamalia, Marlène y Bereiter, Carl (1986)."Research on written composition in Research"in M.C. Wittrock et M. Amarel (eds) Handbook of Research on teaching, New York: Macmillan.

Vandendorpe, Christian (1995)"Au-delà de la phrase: la grammaire du texte"dans Pour un nouvel enseignement de la grammaire, collectif sous la direction de S. Chartrand. Montréal: Éditions Logiques.

Versini (1996). Ordinateur et pédagogie différenciée. Paris: Nathan.

Dirección del sitio (escrito en francés): http: //www.gricea.umontreal.ca

El codigo para:

1. el profesor (antés de hacer escribir sus alumnos): « conte » y « prof007 »

2. los alumnos (después que el profesor ha entrado su nombre y el de su grupo): «conte » y « eleve». 


\section{Primera ficha de revisión}

\section{¿CÓMO DESIGNAR AL PERSONAJE PRINCIPAL?}

Su apellido y/o su nombre $\mathrm{Su}$ origen

Su edad

Sus características

Sus vínculos de parentesco Sus otros vínculos

Sus tareas

Lo que le sucede Cómo reacciona

\author{
Azalaïs \\ la borgoñona \\ la joven, etc. \\ la curiosa, la entusiasta, la rubia, etc.
}

la hermana de Guilhem. la esposa de Arnaud, etc. la amiga de Mahaut, nuestra heroína, nuestro personaje, etc.

la propietaria del castillo, la viajante, la administradora, etc.

la victima, la viuda, la desdichada, la prisionera, etc. la luchadora, la resistente, la tenaz, la prudente, etc.

\section{Secunda ficha de revisión}

\section{¿CÓMO EVITAR LOS TERMINOS VULGARES?}

Elegir y utilizar, por favor, sustitutos sinónimos de lindo, grande, gordo, etc.

Lindo: Agradable, hermoso, deslumbrante, encantador, armonioso, imponente, perfecto, encantador, simpático, espléndido, etc.

Grande: Monstruoso, colosal, gigantesco, esbelto, alto, inmenso, importante, imponente, espacioso, vasto, enorme, excepcional, etc

Gordo: Obeso, grueso, corpulento, robusto, fornido, voluminoso, abultado, pesado, orondo, etc.

Pequeño: Diminuto, menudo, delicado, tenue, grácil, microscópico, invisible, inapreciable, miniatura, bajo, mínimo, exiguo, corto, infinitésimo, estrecho, etc. 
Decir: Advertir, declarar, enunciar, exponer, expresar, indicar, señalar, narrar, nombrar, pronunciar, contar, comentar, detallar, mencionar, citar, etc.

Demandar: Exigir, desear, implorar, insistir, interpelar, interrogar, ordenar, suplica, cuestionar, reclamar, revindicar, querer, etc

Responder: Decir, replicar, objetar, afirmar, asegurar, certificar, atestar, confirmar, declarar, garantizar, prometer, etc.

Ver: Percibir, considerar, contemplar, descubrir, distinguir, examinar, observar, percibir, mirar, remarcar, inspeccionar, etc. 